\title{
AUTOIMMUNITY
}

\section{The threat within}

A recent study published in Cell has shown that $3^{\prime}$ repair exonuclease 1 (TREX1) is essential for preventing cell-intrinsic autoimmunity. Although it has been established that loss-of-function mutations in TREX1 cause the autoimmune conditions Aicardi-Goutieres syndrome and chilblain lupus, the mechanism that underlies the immunopathology of these diseases was previously unknown. Now, Stetson et al. describe how the inefficient breakdown of endogenous retroviral DNA can lead to severe and fatal autoimmunity.

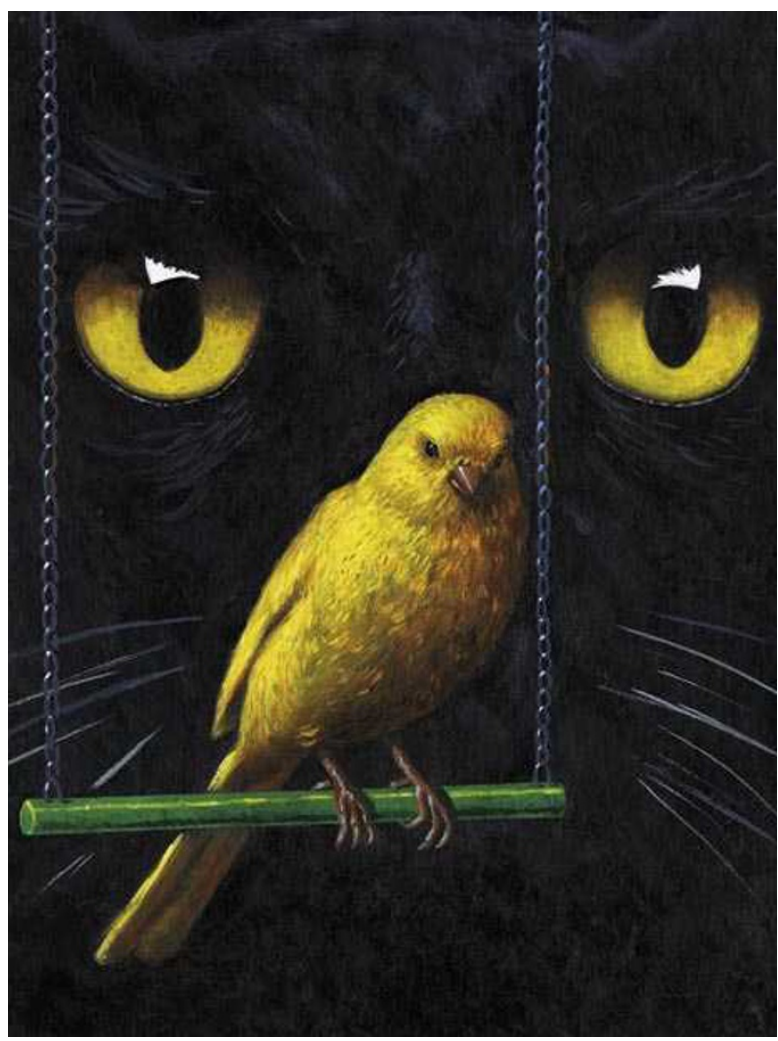

An antiviral response is induced in immune cells following the recognition of viral DNA through the activation of several different pathways, which results in the activation of interferon-regulatory factors (IRFs). Recognition of self DNA can also trigger these pathways, and their overactivation owing to the defective clearance of self nucleic acids has been linked to autoimmunity. In this study, the authors initially set out to identify the proteins that are involved in the interferon-stimulatory DNA (ISD) pathway, a system for sensing cytosolic DNA that is analogous to the RIG-I (retinoic-acid-inducible gene I) pathway for sensing cytosolic RNA. TREX1 was markedly upregulated in response to ISD stimulation, although it did not act directly as a DNA sensor, which indicated that TREX1 might be an important regulator of the ISD pathway.

Investigation of TREX1-deficient mice showed that lethal autoimmunity in these animals was dependent on IRF3, a downstream transcription factor of the ISD pathway. Furthermore, the immunopathology in TREX1deficient mice required lymphocytes and correlated with high levels of circulating autoantibodies. These data suggest that TREX1 is an important negative regulator of the ISD pathway that, when left unchecked, leads to the IRF3-dependent activation of autoreactive lymphocytes.

But what is the mechanism by which the repair exonuclease activity of TREX1 limits autoimmunity? When the authors analysed the inflamed cardiac tissue of TREX1deficient mice, they found a marked increase in the quantity of DNA fragments that were derived from endogenous retroviruses compared with the cardiac tissue of wild-type mice. Further experiments showed that the activity of TREX1 was specific for retroviral but not plasmid DNA and that TREX1 had the capacity to directly metabolize retroviral DNA in an in vitro system.

Because the human genome contains at least 100-fold more retroviral elements than coding genes, mechanisms that metabolize reverse-transcribed endogenous retroviral DNA are crucial to prevent its accumulation and subsequent recognition by the immune system. This study shows that a breakdown in these mechanisms can contribute to autoimmune activation, which provides an explanation for the lethal immunopathology that is observed in patients with AicardiGoutieres syndrome, and indicates that other autoimmune diseases with which TREX1 mutations are associated might also involve defects in the metabolism of self DNA. Finally, these data may also help to define how pathogenic retroviruses are sensed by the host immune system, as the mechanisms involved in their recognition are currently not well understood.

Sarah Allan

ORIGINAL RESEARCH PAPER Stetson, D. B., Ko, J. S., Heidmann, T. \& Medzhitov, R. Trex1 prevents cell-intrinsic initiation of autoimmunity. Cell 134, 587-598 (2008) 